\title{
The radiation impedance of orbiting conductors
}

\author{
J.R. Sanmartín \\ Escuela Técnica Superior de Ingenieros Aeronáuticos, Universidad Politécnica, Madrid
}

M. Martínez-Sánchez

Department of Aeronautics and Astronautics, Massachusetts Institute of Technology, Cambridge

\begin{abstract}
The dispersion relation for waves in a cold, magnetized plasma is discussed using the potential for the longitudinal part of the electric field. This clarifies wave emission from a conductor in low Earth orbit and should be useful in considering the far field and both hot plasma and nonlinear, near-field effects. General formulas for radiation impedance are directly obtained. For tethers a fundamental dependence on contactor size is discussed. Spherical and ellipsoidal contactors and an (anodeless) bare tether are considered. Simple arguments on nonlinear contactor effects lead to a surprisingly simple result for impedances off the Alfvén branch.
\end{abstract}

\section{Introduction}

In a seminal paper, Drell et al. [1965] showed how an orbiting conductor, if in electrical contact with the ionosphere, would excite Alfvén waves. Since the enf induced by the geomagnetic field is proportional to the perpendicular conductor length, using a long space tether, with contaclor ends, would enhance radiation [Banks et al., 1981]. A quantity of interest for both power generation and Alfvén (and higher frequency) signal propagation is the wave impedance [Rasmussen et al., 1985; Dobrowolny and Veltrt, 1986]. Fundamental results were obtained by Barnelt and Olbert [1986] and Estes [1988].

Further work on the impedance was carried out by Donohue et al. [1991] and by Hastengs and Wang [1987] and Hastings et al. [1988], who extended the analysis to conductors carrying ac currents. Fistes [1988] and vom Sterm and Neubauer [1992] studied the field near a tether, Rasmussen et al. [1990] and McKenzie [1991] studied the far field, and Hastıngs and Wang [1989] and vom Sterm and Neubauer [1992] studied two-ion effects. Tether radiation has been modeled in the laboratory [Urrutia and Stenzel, 1989; Stenzel and Urrutia, 1990].

Here, contrary to published analyses, we do not di rectly study wave emission. Instead, we first collect convenient results from the well-known dispersion relation for a cold, magnetized plasma and then solve the wave equation in terms of the potential $\phi$ for the longitudinal part of the electric field of the wave. Only then are conditions particular to orbiting conductors considered; this corrects or clarifies results found in the literature.

Copyright 1995 by the American Geophysical Union.
The $\phi$ formalism, a powerful tool for studying nonlinear or hot-plasma effects, is used here to directly derive complete impedance formulas (sections 2 and 3 ).

For tethers the impedance for branches other than Alfvén is shown to depend heavily on the model for the cathodic and anodic contactors at the ends. Taking into account a nonvanishing contactor length along the tether proves essential for a proper model and yields an impedance varying as the inverse of contactor area. Simple results are obtained for spherical (and ellipsoidal) contactors and for an anodeless bare tether, proposed as an efficient anodic contactor [Sanmartín et al., 1993]. Nonlinear contactor effects suggest an impedance varying as the inverse of current (sections 4 and 5 ).

\section{Wave Emission}

We first recall three well-known results from the usual wave equation for the Fourier-transformed electric field $\mathbf{E}(\mathbf{k}, \omega)$ in a two-component, cold plasma [Akhezer et al., 1975],

$$
-\frac{\mathbf{k} \wedge(\mathbf{k} \wedge \mathbf{E})}{k^{2}}-\frac{\epsilon_{c} \cdot \mathbf{E}}{n^{2}}=\frac{4 \pi i \mathbf{j}_{s}}{\omega n^{2}} .
$$

Here $\mathbf{j}_{s}$ is the source current density, $\mathbf{k}$ is the wave vector, $n \equiv c k / \omega$ is the refractive index, and $\varepsilon_{c}(\omega)$ is the dielectric tensor (with $z$ axis along the field $\mathbf{B}_{0}$ ),

$$
\begin{gathered}
\epsilon_{c}=\left(\begin{array}{lll}
\epsilon_{1} & \imath \epsilon_{2} & 0 \\
-i \epsilon_{2} & \epsilon_{1} & 0 \\
0 & 0 & \epsilon_{3}
\end{array}\right), \epsilon_{3} \simeq 1-\frac{\omega_{p e}^{2}}{\omega^{2}}, \\
\epsilon_{1}=\frac{\omega^{2}-\omega_{\mathrm{UH}}^{2}}{\omega^{2}-\Omega_{e}^{2}} \frac{\omega^{2}-\omega_{\mathrm{LH}}^{2}}{\omega^{2}-\Omega_{\imath}^{2}}, \quad \epsilon_{2} \simeq \frac{\omega_{p e}^{2}}{\omega^{2}-\Omega_{e}^{2}} \frac{\Omega_{e} \omega}{\omega^{2}-\Omega_{\imath}^{2}},
\end{gathered}
$$

with $\Omega_{e}>0, \omega_{\mathrm{UH}}^{2} \simeq \omega_{p e}^{2}+\Omega_{e}^{2}, \omega_{\mathrm{LH}}^{2} \simeq \Omega_{e}^{2}\left(\Omega_{\imath}^{2}+\right.$ $\left.\omega_{p z}^{2}\right) /\left(\Omega_{e}^{2}+\omega_{p e}^{2}\right)$. Here $\omega_{p e}, \omega_{p q}$ and $\Omega_{e}, \Omega_{\imath}$ are plasma 


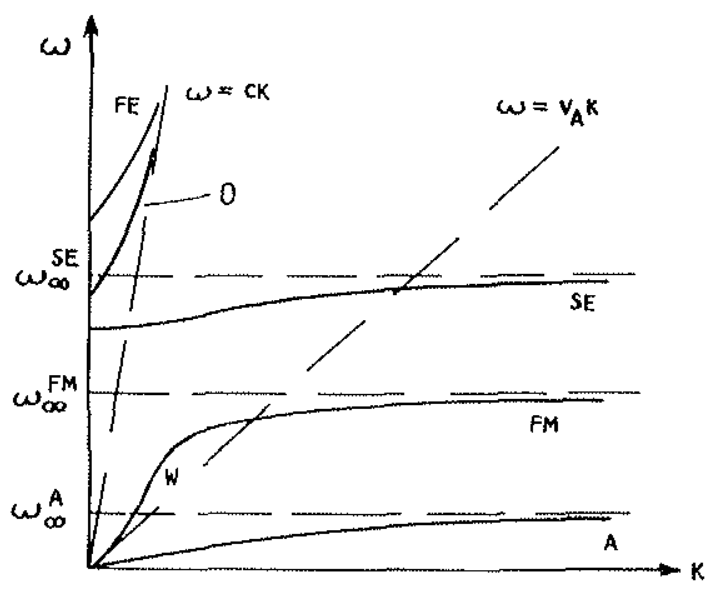

Figure 1. Schematics of branches (fast cxtraordinary (FE), ordinary $(\mathrm{O})$, slow extraordinary (SE), fast magnetosonic (FM), and Alfvén (A)) for the dispersion relation of a cold, magnetized plasma; $W$ is the whistler region in branch $\mathrm{FM}$. The figure is dependent on the angle between $\mathbf{k}$ and the ambient field.

frequencies and gyrofrequencies for electrons and ions; $\omega_{\text {UH }}, \omega_{\mathrm{L}} \mathrm{H}$ are upper and lower hybrid frequencies. (1) Equation (1) yields the Astrom dispersion relation, here written as

$$
\begin{aligned}
& \left(\epsilon_{1} \sin ^{2} \theta+\epsilon_{3} \cos ^{2} \theta-\frac{c_{3} c_{3}}{n^{2}}\right)\left(1-\frac{c_{1}}{n^{2}}\right) \\
& +\left(\sin ^{2} \theta-\frac{c_{3}}{n^{2}}\right) \frac{c_{2}^{2}}{n^{2}}=D(k, \theta, \omega)=0
\end{aligned}
$$

$\theta$ being the angle between $\mathbf{k}$ and $\mathbf{B}_{0}$ There are two branches for $n^{2}(\theta, \omega)$ but there are five branches for $\omega^{2}(k, \theta)$, shown schematically in Figure 1 , as follows: fast extraordinary (FE), ordinary $(\mathrm{O})$, slow extraordinary (SE), fast magnetosonic or compressional Alfvén (FM), and Alfvén or shear Alfvén (A). (2) FE and $O$ branches have $n<1$. (3) SE, FM, and $A$ branches have asymptotes (resonances) $\omega_{\infty}^{\mathrm{SE}}(\theta), \omega_{\infty}^{\mathrm{FM}}(\theta)$, and $\omega_{\infty}^{\mathrm{A}}(\theta)$, obtaincd from (2) in the limit $k \rightarrow \infty$ (that is, $n \rightarrow \infty$ or $\left.\left|\epsilon_{3}\right| / n^{2} \rightarrow 0, j=1-3\right)$, yielding

$$
c_{1} \sin ^{2} \theta+c_{3} \cos ^{2} \theta \equiv D(\infty, \theta, \omega)=0 .
$$

We add here two more points. (1) On the $A$ branch the limit $n \rightarrow \infty$ may be obtained from condition $\omega \rightarrow$ 0 , instead of condition $k \rightarrow \infty$. 'Then, one would also have $\left|c_{3}\right| \rightarrow \infty$, and the ratio $c_{3} / n^{2}$ could take any value. If $c_{1} / n^{2}, \epsilon_{2} / n^{2}$ are small but $c_{3} / n^{2}$ is large, (2) becomes

$$
\epsilon_{3} \cos ^{2} \theta-\frac{\epsilon_{3} \epsilon_{1}}{n^{2}} \equiv D_{\mathrm{A}}(k, \theta, \omega) \simeq 0, \cos ^{2} \theta \ll 1 .
$$

(2) Introducing longitudinal $l$ and transverse $t$ parts of the field,

$$
\mathbf{E}_{l} \equiv \mathbf{k} \mathbf{k} \cdot \mathbf{E} / k^{2}, \quad \mathbf{E}_{t}=\mathbf{E}-\mathbf{E}_{l},
$$

(1) becomes, in all generality,

$$
\mathbf{E}_{t}-\frac{\epsilon_{c}}{n^{2}} \cdot\left(\mathbf{E}_{t}+\mathbf{E}_{l}\right)=\frac{4 \pi i \mathbf{j}_{s}}{\omega n^{2}},
$$

yielding the $\mathbf{E}_{t}$ components along $z$ and perpendicular 1 to $\mathbf{B}_{0}$,

$$
\begin{gathered}
E_{t z}=\frac{c_{3} E_{l z}}{n^{2}-c_{3}}+\frac{4 \pi \imath j_{s z}}{\omega\left(n^{2}-\epsilon_{3}\right)^{\prime}} \\
\mathbf{E}_{t \perp}=\frac{H^{-1} \cdot c_{\perp} \cdot \mathbf{E}_{l \perp}}{n^{2}}+\frac{4 \pi \mathbf{i}}{\omega n^{2}} I^{-1} \cdot \mathbf{j}_{s \perp} \\
c_{\perp}=\left(\begin{array}{cc}
c_{1} & \imath c_{2} \\
-i \epsilon_{2} & \epsilon_{1}
\end{array}\right), \quad H=I-\frac{c_{\perp}}{n^{2}}
\end{gathered}
$$

with $I$ the two-dimensional unit tensor. Use of

$$
\mathbf{k} \cdot \mathbf{E}_{\imath}=0, \quad \mathbf{E}_{l} \equiv-\imath \mathbf{k} \phi
$$

gives an explicit scalar cquation for $\phi$,

$$
\begin{aligned}
& k^{2} D(k, \theta, \omega) \phi=\left[\left(1-\frac{c_{1}}{n^{2}}\right)^{2}-\frac{c_{2}^{2}}{n^{4}}\right] \frac{4 \pi}{\omega} \\
& \times\left[k_{z} j_{s z}+\left(1-\frac{c_{3}}{n^{2}}\right) \mathbf{k}_{\perp} \cdot H^{-1} \cdot \mathbf{j}_{s \perp}\right] .
\end{aligned}
$$

Equation (1) then becomes

$$
k^{2} D(\infty, \theta, \omega) \phi=\frac{4 \pi}{\omega} \mathbf{k} \quad \mathbf{j}_{s}
$$

for conditions leading to $\left(2^{\prime}\right)$, and

$$
k^{2} D_{\Lambda}(k, \theta, \omega) \phi=-\frac{4 \pi}{\omega} \frac{c_{3}}{n^{2}} \mathbf{k}_{\perp} \cdot \dot{j}_{s \perp} \simeq \frac{\omega_{p e}^{2}}{c^{2} k_{\perp}^{2}} \frac{1 \pi}{\omega} \mathbf{k} \cdot \mathbf{j}_{s}
$$

for conditions leading to (2"); we used $\cos ^{2} \theta \ll 1$ to write $\mathbf{k}_{\perp} \cdot \mathbf{j}_{s \perp} \simeq \mathbf{k} \cdot \mathbf{j}_{s}$.

We are now in a position to discuss wave emission from an orbiting conductor sustaining a steady current. In the terrestrial reference frame the Doppler relation reads

$$
\omega=k_{r} V
$$

as usual, we take $\mathbf{B}_{0}$ and orbital velocity $\mathbf{V}$ horizontal and perpendicular to each other, with $x$ axis along V. In the ionosphere, the Alfvén velocity $V_{A} \equiv c \Omega_{2} / \omega_{p q}$ satisfics the conditions [Barnett and Olbert, 1986] (hereinafter referred to as $\mathrm{BO}$ )

$$
\begin{gathered}
V \ll V_{\mathrm{A}} \ll c, \\
\Omega_{\mathrm{e}}^{2} \ll \omega_{\mathrm{pe}}^{2} \text { or } V_{\mathrm{A}}^{2} \ll c^{2} m_{\mathrm{e}} / m_{2}
\end{gathered}
$$

$\left(V \simeq 7 \mathrm{~km} / \mathrm{s}, V_{\mathrm{A}} \sim 300 \mathrm{~km} / \mathrm{s}, m_{\imath} / m_{e} \simeq 30.000\right)$. It also satisfies the condition

$$
V_{A}^{2} \ll V^{2} m_{\imath} / m_{e}
$$


$m_{\ell} / m_{e}$ is the ion-electron mass ratio. The lower-hybrid frequency is now $\omega_{L H} \simeq\left(\Omega_{e} \Omega_{2}\right)^{1 / 2}$.

Note from (5) and (6a) that $n$ is here very large,

$$
1 / n \equiv \omega / c k=k_{x} V / k c \ll 1 .
$$

This means, first, that no FE or $\mathrm{O}$ emission is possible. It suggests, secondly, that (2') might apply to the SE and FM branches, which is indeed the case. For SE, use of $\omega_{\infty}^{\mathrm{SE}}(\theta)$ [Akhıezer et al, 1975$]$,

$$
\omega_{p e} \leq \omega_{\infty}^{\mathrm{SE}} \simeq\left(\omega_{p e}^{2}+\Omega_{e}^{2} \sin ^{2} \theta\right)^{1 / 2} \leq \omega_{\mathrm{UH}},
$$

gives $\epsilon_{2} \simeq \Omega_{e} / \omega_{p e}, \epsilon_{1} \simeq-c_{2}^{2} \cos ^{2} \theta, \epsilon_{3} \simeq \epsilon_{2}^{2} \sin ^{2} \theta$; condition (7) (and (6b)) then shows all $\epsilon_{\jmath} / n^{2}$ to be small. Alternatively, one can find $\left(\omega_{\infty}^{\mathrm{SE}}-\omega\right) / \omega \ll 1$, directly from (2), for $\Omega_{e} / c k$ small as in the present case $\left(\Omega_{e} / c k \simeq \epsilon_{2} / n\right)$. For FM, use of $\omega_{\infty}^{\mathrm{FM}}(\theta)[$ Akhezer et al., 1975],

$$
\omega_{\mathrm{LH}} \leq \omega_{\infty}^{\mathrm{FM}} \simeq\left(\Omega_{e}^{2} \cos ^{2} \theta+\omega_{\mathrm{LK}}^{2} \sin ^{2} \theta\right)^{1 / 2} \leq \Omega_{\mathrm{e}},
$$

similarly proves that $\left|\epsilon_{j}\right| / n^{2}(\jmath=1-3)$ and $\left(\omega_{\infty}^{\mathrm{FM}}-\right.$ $\omega) / \omega$ are small for $\omega_{\mathrm{LH}} / V_{\mathrm{A}} k_{\perp} \ll 1$. Herc $\omega_{\mathrm{LH}} / V_{\mathrm{A}} k_{\perp} \equiv$ $\omega_{L H} V k_{x} / \omega V_{\mathrm{A}} k_{\perp}$ is indeed small because of condition $V / V_{\mathrm{A}} \ll 1$.

The large refractive index finally suggests that (2') or (2"), or an intermediate dispersion relation, might apply to the $\mathrm{A}$ branch. Here we have

$$
\frac{\left|\epsilon_{3}\right|}{n^{2}} \simeq \frac{\omega_{\mathrm{pe}}^{2}}{c^{2} k^{2}}=\frac{\Omega_{2}^{2}}{\omega^{2}} \frac{k_{x}^{2}}{k^{2}} \frac{m_{\imath} V^{2}}{m_{e} V_{\mathrm{A}}^{2}} .
$$

Since this branch always has $\omega \leq \Omega_{z}$, condition (6c) makes $\left|\epsilon_{j}\right| / n^{2}$ large and (2") valid, except for $k_{y} / k_{x}$ large enough. The equation $D_{\mathrm{A}}=0$ has the root

$$
\omega_{\mathrm{A}}\left(k_{z}\right) \equiv \Omega_{\mathrm{z}} V_{\mathrm{A}} k_{z} /\left(\Omega_{\mathrm{z}}^{2}+V_{A}^{2} k_{z}^{2}\right)^{1 / 2} .
$$

Note that $\cos \theta \equiv k_{z} / k=\left(k_{x} V / k V_{\mathrm{A}}\right) V_{\mathrm{A}} k_{z} / \omega_{\mathrm{A}}$ is small as required, unless $\omega$ is very close to $\Omega_{i}$. We now make the ansatz that negligible energy is carried by such frequencies and by large $k_{y} / k_{x}$ values, which may thus be ignored. Note also that $c_{1} / n^{2}\left(=\cos ^{2} \theta\right)$ and $c_{2} / n^{2}\left(\simeq \epsilon_{1} \omega / n^{2} \Omega_{t}\right)$ are small as assumed.

For both the SE and FM modes, (2'), (3), and (4') give

$$
\phi=\frac{4 \pi \mathbf{k} \cdot \mathbf{j}_{s}}{\omega\left(\epsilon_{1} k_{\perp}^{2}+\epsilon_{3} k_{z}^{2}\right)}, \quad \mathbf{E} \simeq \mathbf{E}_{l}=-\imath \mathbf{k} \phi
$$

with $\mathbf{E}_{t}=0\left(n^{-2}\right)$. For the Alfvén mode, (2"), (3), and (4") give

$$
\phi=\frac{4 \pi \mathbf{k} \cdot \mathbf{j}_{s}}{\omega\left(\epsilon_{1} k_{\perp}^{2}-n^{2} k_{z}^{2}\right)}, \quad \mathbf{E} \simeq \mathbf{E}_{l \perp}=-i \mathbf{k}_{\perp} \phi
$$

with $\mathbf{E}_{t z} \simeq-\mathbf{E}_{l z}, \mathbf{E}_{t \perp}=0\left(n^{-2}\right)$. Since $\omega_{\infty}$ is a function of just $\theta$, or $k_{z} / k, \mathrm{SE}$ and $\mathrm{FM}$ waves have a group velocity perpendicular to $\mathbf{k}$ in the $\mathbf{B}_{0}, \mathbf{k}$ plane, a fact not entirely clear in the literature [BO;Hastings and Wang, 1987]. Since $\omega_{\mathrm{A}}$ depends only on $k_{z}$, A waves have group velocity along $\mathbf{B}_{0}$, with $\mathbf{k}$ nearly perpendicular. Note that certain results in BO's analysis (figures $2 \mathrm{~b}$ and $2 \mathrm{c}$, for bands II and III, of that paper) represent just the well-known formulas (8a) and (8b) (figure 2a, for band I, of BO's paper represents $\omega_{\mathrm{A}}\left(k_{x} / k_{z}\right)$ from (9), with $\omega_{\mathrm{A}}$ written as $V k_{x}$ where necessary).

Note also that there is no whistler emission (part W on the FM branch of Figure 1); Stenzel and Urrutia's experiments showing whistlers do not apply to an ionospheric tether; they either fail to reproduce the steady condition $\omega=k_{x} V$ [Urrutia and Stenzel, 1989] or correspond to the opposite regime $V \simeq 2 \times 10^{7} \mathrm{~cm} / \mathrm{s} \gg$ $V_{\mathrm{A}} \simeq 4 \times 10^{5} \mathrm{~cm} / \mathrm{s}$ [Stenzel and Urrutia, 1990]. Radiation occurs at the contactors, where $\nabla \cdot \mathbf{j}_{s} \neq 0$, because it depends on the source divergence $\mathbf{k} \cdot \mathbf{j}_{s}$, a fact first noticed by Estes [1988] and clearly arising from the quasi-electrostatic character of the field, $\mathbf{E} \simeq-i \mathbf{k} \phi$ or $-\imath \mathbf{k}_{\perp} \phi$. For FM waves this followed from condition $V \ll V_{\mathbf{A}} ; \mathbf{F M}$ emission along a tether, as found by Stenzel and Urrutia [1990], corresponds to the opposite, whistler regime, $V \gg V_{\mathrm{A}}$. Note, however, that condition $V \ll V_{\mathrm{A}}$, being dependent on planetary parameters (surface gravity, radius, ionopheric density, and ambient field $\mathbf{B}_{0}$ ), is not an intrinsic property of tethers; tether radiation might thus depend, in general, on current source features other than its divergence [Donohue et al.,1991].

In the following sections we shall use the present $\phi$ formalism to determine radiation impedance formulas. The formalism, however, should also be useful in studying (1) how to match linear results for the field to nonlinear results near the tether, where $\mathbf{E}$ should be nearly electrostatic; (2) the radiation pattern in the transverse far field of the waves, given clearly as

$$
\mathbf{E}_{t} \simeq-i \frac{\epsilon_{c} \cdot \mathbf{k}}{n^{2}} \phi,(\mathrm{SE}, \mathrm{FM}) \quad \text { or } \quad \mathbf{E}_{t} \simeq i k_{z} \phi \mathbf{1}_{z}
$$

(3) hot plasma effects [(10a) would still hold, with the hot dielectric tensor $c_{h}$ replacing $\epsilon_{c}$ in the expression $\left.\epsilon_{1} k_{\perp}^{2}+\epsilon_{3} k_{z}^{2} \equiv \mathbf{k} \cdot \epsilon_{c} \cdot \mathbf{k}\right]$.

\section{The Impedance Formulas}

To determine the impedance, there is no need to compute the near field [Estes, 1988] or the far field [Barnett and Olbert, 1986; Hastings et al., 1988]. For SE or FM waves we have $\mathbf{E}(\mathbf{r}, t) \simeq-\nabla \phi(\mathbf{r}, t)$ in space-time coordinates $\mathbf{r}, t$. Since $\mathbf{j}_{s}$ vanishes outside certain volume, we find a radiated power 


$$
\begin{gathered}
\mathbf{P}_{\text {rad }}=-\int \mathbf{j}_{s} \cdot \mathbf{E} d \mathbf{r}=-\int \phi \nabla \cdot \mathbf{j}_{s} d \mathbf{r} \\
=-\int d \mathbf{r} \int \frac{d \mathbf{k}_{1} d \omega_{1}}{4 \pi^{2}} e^{i \mathbf{k}_{1} \cdot \mathbf{r}-i \omega_{1} t} \phi\left(\mathbf{k}_{1}, \omega_{1}\right) \\
\times \int \frac{d \mathbf{k} d \omega}{4 \pi^{2}} e^{i \mathbf{k} \cdot \mathbf{x}-i \omega t} i \mathbf{k} \cdot \mathbf{j}_{s}(\mathbf{k}, \omega)
\end{gathered}
$$

with $\phi(\mathbf{k}, \omega)$ given by $(10 \mathrm{a})$. For $\mathrm{A}$ waves we have $\mathbf{E} \simeq$ $-\nabla_{\perp} \phi$ and, again setting $\mathbf{k}_{\perp} \cdot \mathbf{j}_{\boldsymbol{1}} \simeq \mathbf{k} \cdot \mathbf{j}_{8}$, we arrive back at (11) with $\phi(\mathbf{k}, \omega)$ given by $(10 b)$. The $\mathbf{r}$ integral is immediate, $\int d \mathbf{r} \exp \left[i\left(\mathbf{k}_{1}+\mathbf{k}\right) \cdot \mathbf{r}\right]=8 \pi^{3} \delta\left(\mathbf{k}_{1}+\mathbf{k}\right)$. This makes the $\mathbf{k}_{1}$ integral immediate too.

The source divergence can be written as

$$
\begin{gathered}
i \mathbf{k} \cdot \mathbf{j}_{s}=\int \frac{d \mathbf{r} d t}{4 \pi^{2}} e^{-\mathbf{k} \mathbf{k} \cdot \mathbf{r}+i \omega t} \nabla \cdot \mathbf{j}_{s}(x-V t, y, z) \\
\equiv i I_{s} \delta\left(\omega-k_{x} V\right) g(\mathbf{k})
\end{gathered}
$$

We have set $x-V t \rightarrow x$ and introduced the current $I_{s}$ in the conductor and a dimensionless function $g(\mathbf{k})$ defined by

$$
g(\mathbf{k}) \equiv-i \int d \mathbf{r} \nabla \cdot \mathbf{j}_{s}(\mathbf{r}) \exp (-i \mathbf{k} \cdot \mathbf{x}) / 2 \pi I_{s}
$$

The $\omega_{1}$ and $\omega$ integrals in (11) are now straightforward, yielding the impedance, $Z \equiv$ Power $/ I_{s}^{2}$, as

$$
\begin{gathered}
Z=\int \frac{2 i|g(\mathbf{k})|^{2} d \mathbf{k}}{\omega k_{\perp}^{2}}\left[\epsilon_{1}(\omega)\right. \\
\left.+k_{z}^{2}\left\{\begin{array}{c}
-c^{2} / \omega^{2} \\
\epsilon_{3}(\omega) / k_{\perp}^{2}
\end{array}\right\}\right]^{-1}, \quad\left\{\begin{array}{l}
\mathrm{A} \\
\mathrm{SE}, \mathrm{FM}
\end{array}\right\}
\end{gathered}
$$

with $\omega=k_{x} V$; we used the relation $g(-\mathbf{k})=-g^{*}(\mathbf{k})$.

\section{Alfven Mode}

For the $\mathrm{A}$ branch we have $\epsilon_{1} \simeq \omega_{p i}^{2} /\left(\Omega_{i}-\omega^{2}\right)$ and

$$
\begin{gathered}
\epsilon_{1}-\frac{c^{2} k_{z}^{2}}{\omega^{2}}=\frac{c^{2}}{V_{\mathrm{A}}^{2}} \frac{\Omega_{z}^{2}+V_{\mathrm{A}}^{2} k_{z}^{2}}{\Omega_{i}^{2}-V^{2} k_{x}^{2}} \\
\times \frac{\left(k_{x}+i \Delta\right)^{2}-\left[\omega_{\mathrm{A}}\left(k_{z}\right) / V\right]^{2}}{k_{x}^{2}}, \Delta \rightarrow 0^{+},
\end{gathered}
$$

where we set $\omega \rightarrow \omega+i \nu \quad\left(\nu \rightarrow 0^{+}\right)$, which is the usual rule for wave poles, arising from considerations of weak collisions, adiabatic switching in the remote past, or the radiation condition at infinity. Note that the integrand in (13) is even in $k_{y}, k_{z}$, and odd in $k_{x}$ (for $\Delta$ strictly zero). Then we find

$$
\begin{aligned}
& Z_{\mathrm{A}}=\int_{0}^{\infty} d k_{y} \int_{0}^{\infty} d k_{z} \int_{0}^{\infty} \frac{d k_{x}\left(\Omega_{i}^{2}-V^{2} k_{x}^{2}\right)|g|^{2}}{c^{2} V k_{\perp}^{2}\left(\Omega_{z}^{2}+V_{\mathrm{A}}^{2} k_{z}^{2}\right)} \\
& \times\left[\frac{8 i V_{\mathrm{A}}^{2} k_{z}}{\left(k_{x}+i \Delta\right)^{2}-\omega_{\mathrm{A}}^{2} / V^{2}}-\frac{8 i V_{\mathrm{A}}^{2} k_{x}}{\left(k_{x}-i \Delta\right)^{2}-\omega_{\mathrm{A}}^{2} / V^{2}}\right] \\
& =\frac{8 \pi V_{\mathrm{A}}^{2}}{c^{2} V} \int_{0}^{\infty} \int_{0}^{\infty} \frac{d k_{y} d k_{z} \Omega_{i}^{4}}{\left(\Omega_{z}^{2}+V_{\mathrm{A}}^{2} k_{z}^{2}\right)^{2}} \frac{\left|g\left(\omega_{\mathbf{A}} / V_{1} k_{y}, k_{z}\right)\right|^{2}}{k_{y}^{2}+\omega_{\mathrm{A}}^{2} / V^{2}}
\end{aligned}
$$

where we used

$\frac{\Delta}{\left(k_{x}-\omega_{\mathbf{A}} / V\right)^{2}+\Delta^{2}} \rightarrow \pi \delta\left(k_{x}-\frac{\omega_{A}}{V}\right)$ as $\Delta \rightarrow 0^{+}$.

A formula somewhat simpler than (14) is obtained by changing variables in the double integral from $\left(k_{y}, k_{x}\right)$ to $\left(k_{y}, \omega_{\mathrm{A}} / V\right)$, i.e., effectively changing to $\left(k_{y}, k_{x}\right)$. The result can thus be directly obtained from (13) by carrying out the integration over $k_{z}$. We find

$$
\begin{gathered}
\epsilon_{1}-\frac{c^{2} k_{z}^{2}}{\omega^{2}}=-\frac{c^{2}}{V^{2}} \frac{k_{z}^{2}-\left[k_{\mathrm{A}}^{*}\left(k_{x}+i \Delta\right)\right]^{2}}{k_{x}^{2}} \\
k_{\mathrm{A}}^{*}\left(k_{x}\right) \equiv \frac{\Omega_{i} k_{x} V / V_{\mathrm{A}}}{\left(\Omega_{i}^{2}-k_{x}^{2} V^{2}\right)^{1 / 2}} \\
Z_{\mathrm{A}}=\int_{0}^{\infty} d k_{y} \int_{0}^{\infty} \frac{V d k_{x}}{c^{2} k_{\perp}^{2}} \int_{-\infty}^{\infty} d k_{z}|g|^{2} \\
\times\left\{\frac{4 i k_{x}}{k_{z}^{2}-\left[k_{\mathrm{A}}^{*}\left(k_{x}-i \Delta\right)\right]^{2}}-\frac{4 i k_{x}}{k_{z}^{2}-\left[k_{\mathrm{A}}^{*}\left(k_{x}+i \Delta\right)\right]^{2}}\right\}
\end{gathered}
$$

Since we have $k_{\mathrm{A}}^{*}\left(k_{x} \pm i \triangle\right)=k_{\mathrm{A}}^{*}\left(k_{x}\right) \pm i \triangle d k_{\mathrm{A}}^{*} / d k_{x}$ with $d k_{\mathrm{A}}^{*} / d k_{x}$ positive (for $k_{x}$ positive), the $k_{z}$ integral yields $8 \pi k_{x}\left|g\left(k_{x}, k_{y}, k_{\mathrm{A}}^{*}\right)\right|^{2} / k_{\mathrm{A}}^{*}$ for $k_{x}$ such that $k_{\mathrm{A}}^{*}\left(k_{x}\right)$ is real $\left(k_{x}<\Omega_{\varepsilon} / V\right)$, and zero otherwise. Defin ing $k_{i} \equiv \Omega_{t} / V$ we arrive at

$$
\begin{aligned}
& Z_{\mathrm{A}}=\frac{8 \pi V_{\mathrm{A}}}{c^{2}} \int_{0}^{k_{i}} \frac{d k_{x}}{k_{i}}\left(k_{i}^{2}-k_{x}^{2}\right)^{1 / 2} \\
& \times \int_{0}^{\infty} \frac{d k_{y}\left|g\left[k_{x}, k_{y}, k_{\mathrm{A}}^{*}\left(k_{x}\right)\right]\right|^{2}}{k_{y}^{2}+k_{x}^{2}} .
\end{aligned}
$$

\section{Slow Extraordinaxy and Fast Magnetosonic Modes}

For the SE branch we have $\epsilon_{3}=\left(\omega^{2}-\omega_{p e}^{2}\right) / \omega^{2}, \epsilon_{1} \simeq$ $-\left(\omega_{\text {UH }}^{2}-\omega^{2}\right) / \omega^{2}$. Proceeding as with Alfvén waves we get

$$
\begin{gathered}
\epsilon_{1}+\frac{k_{z}^{2}}{k_{\perp}^{2}} \epsilon_{3}=\frac{k_{x}^{2}+k_{2 s}^{2}}{k_{x}^{2}+k_{y}^{2}} \frac{\left(k_{x}+i \Delta\right)^{2}-k_{1 s}^{2}}{k_{x}^{2}}, \quad \Delta \rightarrow 0^{+} \\
Z_{\mathrm{SE}}=\frac{8 \pi}{V} \int_{0}^{\infty} d k_{y} \int_{0}^{\infty} d k_{z} \frac{\left|g\left(k_{1 s}, k_{y}, k_{z}\right)\right|^{2}}{k_{2 s}^{2}+k_{1 s}^{2}}
\end{gathered}
$$


where $k_{1 s}, k_{2 s}$ are known functions of $k_{y}, k_{z}$ which we will not pause to give. Changing variables from $k_{y}, k_{z}$ to $k_{y}, k_{1 s}$ (effectively $k_{y}, k_{x}$ ) or directly integrating over $k_{z}$ in (13), we finally find

$$
\begin{aligned}
& Z_{\mathrm{SE}}= \frac{8 \pi}{V} \int_{k_{\mathrm{pe}}}^{k_{\mathrm{UH}}} \frac{k_{x} d k_{x}}{\left(k_{\mathrm{UH}}^{2}-k_{x}^{2}\right)^{1 / 2}\left(k_{x}^{2}-k_{p e}^{2}\right)^{1 / 2}} \\
& \times \int_{0}^{\infty} \frac{d k_{y}\left|g\left(k_{x}, k_{y}, k_{\mathrm{SE}}^{*}\right)\right|^{2}}{\left(k_{x}^{2}+k_{y}^{2}\right)^{1 / 2}} \\
& k_{\mathrm{SE}}^{*} \equiv\left(k_{x}^{2}+k_{y}^{2}\right)^{1 / 2}\left(k_{\mathrm{UH}}^{2}-k_{\mathfrak{x}}^{2}\right)^{1 / 2} /\left(k_{x}^{2}-k_{p e}^{2}\right)^{1 / 2} \\
& k_{p e, \mathrm{UH}} \equiv \omega_{p e, \mathrm{UH}} / V .
\end{aligned}
$$

For the FM branch we have $\epsilon_{3} \simeq-\omega_{p e}^{2} / \omega^{2}, \quad \epsilon_{1} \equiv$ $-\epsilon_{3}\left(\omega^{2}-\omega_{\mathrm{LH}}^{2}\right) /\left(\Omega_{e}^{2}-\omega^{2}\right)$,

$\epsilon_{1}+\frac{k_{z}^{2}}{k_{\perp}^{2}} \epsilon_{3}=\frac{k_{p e}^{2}}{k_{x}^{2}} \frac{k_{x}^{2}+k_{2 \mathrm{~F}}^{2}}{k_{x}^{2}+k_{y}^{2}} \frac{\left(k_{x}+i \Delta\right)^{2}-k_{1 \mathrm{~F}}^{2}}{k_{e}^{2}-k_{x}^{2}}, \quad \Delta \rightarrow 0^{+}$

$Z_{\mathrm{FM}}=\frac{8 \pi}{V} \int_{0}^{\infty} \int_{0}^{\infty} \frac{d k_{y} d k_{z}}{k_{p e}^{2}} \frac{k_{e}^{2}-k_{1 \mathrm{~F}}^{2}}{k_{2 \mathrm{~F}}^{2}+k_{1 \mathrm{~F}}^{2}}\left|g\left(k_{1 \mathrm{~F}}, k_{y}, k_{z}\right)\right|^{2}$

with $k_{e} \equiv \Omega_{e} / V$ and $k_{1 \mathrm{~F}}, k_{2 \mathrm{~F}}$, some given functions of $k_{y}, k_{z}$. Also,

$$
\begin{aligned}
& Z_{\mathrm{FM}}=\frac{8 \pi}{V} \int_{k_{\mathrm{LH}}}^{k_{c}} \frac{k_{x} d k_{x}}{k_{p e}^{2}}\left(\frac{k_{\mathrm{e}}^{2}-k_{x}^{2}}{k_{x}^{2}-k_{\mathrm{LH}}^{2}}\right)^{1 / 2} \\
& \times \int_{0}^{\infty} d k_{y} \frac{\left|g\left(k_{x}, k_{y}, k_{\mathrm{FM}}^{*}\right)\right|^{2}}{\left(k_{x}^{2}+k_{y}^{2}\right)^{1 / 2}} \\
& k_{\mathrm{FM}}^{*} \equiv\left(k_{x}^{2}+k_{y}^{2}\right)^{1 / 2}\left(k_{x}^{2}-k_{\mathrm{LH}}^{2}\right)^{1 / 2} /\left(k_{e}^{2}-k_{x}^{2}\right)^{1 / 2}, \\
& k_{\mathrm{LH}} \equiv \omega_{\mathrm{LH}} / V .
\end{aligned}
$$

BO derived formulas (14')-(16') for a particular source current by determining the far field; formulas (14)-(16) are new.

\section{The Source Divergence of a Tether}

As usual, we will take the $y$ axis along the vertical tether, with velocity $\mathbf{V}$ and field $\mathbf{B}_{0}$ along axes $x$ and $z$, as previously noted (Figure 2). Most current divergences discussed in the literature may then be written as

$$
\nabla \cdot \dot{\mathbf{j}}_{s}(\mathbf{r})=f(x, z) j[\delta(y+L / 2)-\delta(y-L / 2)]
$$

for an upward current and a tether length $L ; f(x, z)$ is a step function equal to $1(0)$ inside (outside) certain cross sections representing equal bottom and top contactors. BO considered a circle of radius $b\left(j=I_{s} / \pi b^{2}\right)$, Hastings

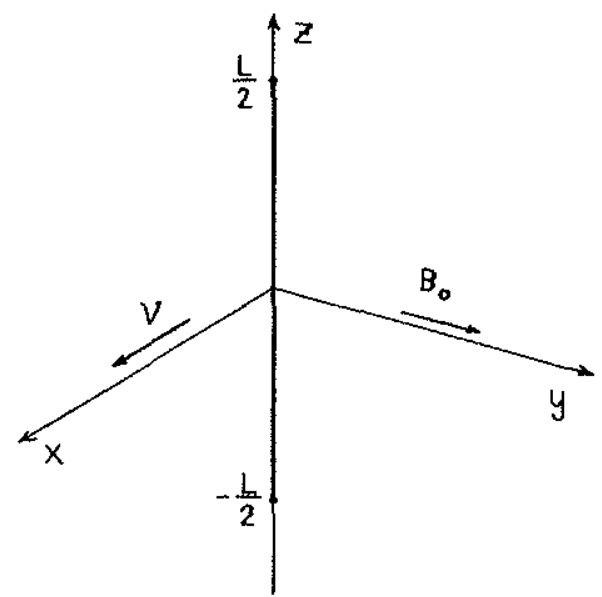

Figure 2. Coordinate system used to compute tether impedance.

and Wang [1987] and Hastings et al. [1988] considered rectangular surfaces of sides $L_{x}, L_{z}\left(j=I_{s} / L_{x} L_{z}\right)$. Estes [1988] source divergence is that of a ribbon of width $L_{x}$, given by (17) with a segment $L_{x}$ along $x$ as cross section, and $j=I_{s} \delta(z) / L_{x}$. An additional case of interest is a ring of radius $R$, with $j=I_{s} \delta\left(r_{\text {per }}-\right.$ $R) / 2 \pi R, r_{\text {per }}=\left(x^{2}+z^{2}\right)^{1 / 2}$ (perpendicular to tether axis).

Using (17) in (12) we find

$$
g(\mathbf{k})=\frac{1}{\pi} \sin \left(k_{y} \frac{L}{2}\right) g_{\mathrm{per}}\left(k_{x}, k_{z}\right)
$$

with $\left(k_{x}, k_{z}\right) \equiv \mathbf{k}_{\mathrm{per}}$, and $\boldsymbol{g}_{\mathrm{per}} \equiv<\exp \left(-i \mathbf{k}_{\mathrm{per}} \cdot \mathbf{r}_{\mathrm{per}}\right)>$ being the average of the exponential in the cross section. For the above models we obtain

$$
\begin{gathered}
g_{\mathrm{per}}^{\mathrm{ring}}=J_{0}\left(k_{\mathrm{per}} R\right), \quad g_{\mathrm{per}}^{\mathrm{Est}}=\frac{\sin \left(k_{x} L_{x} / 2\right)}{k_{x} L_{x} / 2}, \\
g_{\mathrm{per}}^{\mathrm{B} 0}=\frac{J_{1}\left(k_{\mathrm{per}} b\right)}{k_{\mathrm{per}} b / 2}, \quad g_{\mathrm{per}}^{\mathrm{Hast}}=\frac{\sin \left(k_{x} L_{x} / 2\right)}{k_{x} L_{x} / 2} \frac{\sin \left(k_{z} L_{z} / 2\right)}{k_{z} L_{z} / 2}
\end{gathered}
$$

with Est denoting Estes [1988] and Hast denoting Hastings and Wang [1987] and Hastings et al. [1988]. For models deriving from (17), contactors have vanishing characteristic length along the tether (as different from distance $L$ between contactors).

If contactor lengths in the plane $(x, z)$ perpendicular to the tether are small enough, the above models collapse into a common form, with $g_{\text {per }} \rightarrow 1, g \rightarrow$ $\sin \left(k_{y} L / 2\right) / \pi$. One may also consider negligible perpendicular lengths for a general source divergence not given by (17); we may then write $g(\mathbf{k}) \rightarrow g\left(k_{y}\right)$. This will later be of interest for the Alfvén mode of a bare tether.

\section{Alfvén Mode}

In (14') we have $k_{z}=k_{\mathrm{A}}^{*}\left(k_{x}\right) \sim k_{x} V / V_{\mathrm{A}} \ll k_{x}<$ $\Omega_{i} / V(\simeq 1 / 36 \mathrm{~m})$. Thus we may certainly neglect the 
characteristic $z$ length and set $k_{z}=k_{\mathrm{A}}^{*} \simeq 0$ in $g_{p e r}$. Using (18) in (14') we can carry out the $k_{y}$ integration to obtain

$$
\begin{gathered}
Z_{\mathrm{A}} \simeq \frac{2 V_{\mathrm{A}}}{c^{2}} \int_{0}^{k_{i}} \frac{d k_{x}}{k_{x}}\left(1-\frac{k_{x}^{2}}{k_{i}^{2}}\right)^{1 / 2} \\
\times\left(1-e^{-k_{x} L}\right)\left|g_{\mathrm{per}}\left(k_{x}, 0\right)\right|^{2} .
\end{gathered}
$$

BO gave (19) with $g_{\text {per }}^{\text {B0 }}$; Estes [1988] gave a close approximation for his model, using $g_{\text {per }}^{\text {Est }}$ in place of $\left|g_{\text {per }}^{\text {Est }}\right|^{2}$.

If the characteristic length along $x$ may also be neglected, one sets $\left|g_{\text {per }}\right|^{2}=1$ in (19) and, for $L \gg V / \Omega_{i}$, obtains

$$
Z_{\mathrm{A}}=\left(2 V_{\mathrm{A}} / c^{2}\right) \ln \left(2 e^{\gamma-1} \Omega_{i} L / V\right), \quad \gamma \simeq 0.577,
$$

a result found by $\mathrm{BO}$ and by Estes. The dominant, logarithmic contribution comes from frequencies $\omega \sim$ $V / L$. Since $k_{x}$ is $\omega / V$, the single integral over $k_{x}$ in (19) contains the Alfvén power spectrum; clearly, frequencies $\omega \simeq \Omega_{i}$ contribute negligibly to the impedance, proving a previous ansatz.

For an arbitrary source divergence with negligible perpendicular lengths, a new, general formula results from using $g\left(k_{y}\right)$ in $\left(14^{\prime}\right)$ and carrying out the $k_{x}$ integration

$$
Z_{\mathrm{A}}=\frac{4 \pi^{2} V_{\mathrm{A}}}{c^{2}} \int_{0}^{\infty} \frac{d k_{y}}{k_{y}} \frac{\left(k_{i}^{2}+k_{y}^{2}\right)^{1 / 2}-k_{y}}{k_{i}}\left|g\left(k_{y}\right)\right|^{2} .
$$

For $g=\sin \left(k_{y} L / 2\right) / \pi$, and $L k_{i} \gg 1$, one recovers $\left(19^{\prime}\right)$. When deriving (19)-(19") from (14'), one can verify that large values of $k_{y} / k_{x}$ may be ignored, again proving a previous ansatz.

\section{Slow Extraordinary and Fast Magnetosonic Modes}

As later detailed, perpendicular contactor lengths are large compared with $1 / k_{\text {per }}$ for the FM and SE modes. This makes impedances quite dependent on the size of contactors, a case opposite that of A waves, as first observed by BO for FM waves, and repeatedly discussed afterward. Here we note that the effect is heavily dependent on contactor model. At large sizes the factor $|g|^{2}$ in the (15')-(16') integrals, and therefore the impedance itself, scales differently with contactor size for different contactor shapes. We have

$$
\begin{array}{cc}
Z^{\text {ring }} \propto 1 / R, & Z^{\text {Est }} \propto 1 / L_{x}^{2}, \\
Z^{\mathrm{B} 0} \propto 1 / b^{3}, & Z^{\text {Hast }} \propto 1 / L_{x}^{2} L_{z}^{2},
\end{array}
$$

a square sine or cosine averaging to $1 / 2$ inside the integrals (see Appendix).

This sensitivity of impedance to the source divergence model raises the fundamental question of the proper model. We now make two points. First, there is a simple rule applying to the above results; for both the ring and Estes sources, the divergence occurs at a finite, one-dimensional set (line contactors); if the set closes on itself, with no boundary (ring), one has $Z \propto$ (length) $^{-1}$; otherwise [Estes, 1988], one has $Z \propto$ (length) $^{-2}$. Now the $\mathrm{BO}$ and Hastings and Wang [1987] and Hastings et al. [1988] sources use two-dimensional sets (surface contactors), the first having one boundary (radially), the Hastings set having boundaries on both dimensions; one would thus expect scalings $Z^{\mathrm{B} 0} \propto$ (length) $)^{-1} \times$ (length) $)^{-2}$ and $Z^{\text {Hast }} \propto$ (length) $^{-2} \times(\text { length })^{-2}$, in agreement with the above results (see the Appendix).

Secondly, this sensitivity questions the validity of neglecting the contactor characteristic length along the tether, as in all those models. We now show that taking into account that length actually determines the proper model, i.e., the correct dependence of $Z$ on contactor size. Nonvanishing lengths along the tether were considered in studying contactor planar surfaces of different orientations [Hastings et al., 1988], the Alfvén far field [Rasmussen et al., 1990], and Debye sheath effects [Donohue et al., 1991].

In a proper model the current divergence should clearly occur at a surface topologically equivalent to a sphere, e.g., a rotational ellipsoid with axis along $\mathbf{B}_{0}$. This is a finite two-dimensional set as in the models of Hastings and Wang [1987] and Hastings et al. [1988] and $\mathrm{BO}$, but it has no boundaries at all; we would thus expect finding $Z \propto$ (length) $^{-2}$, and this is indeed the case. For instance, for (symmetrical) spherical contactors of radius $R$ the source divergence is

$$
\begin{aligned}
\nabla \cdot \mathbf{j}_{s} & =\frac{I_{s}}{4 \pi R^{2}}\left[\delta\left(\left|\mathbf{x}+\frac{L}{2} \mathbf{1}_{\mathbf{y}}\right|-R\right)\right. \\
& \left.-\delta\left(\left|\mathbf{x}-\frac{L}{2} \mathbf{1}_{y}\right|-R\right)\right]
\end{aligned}
$$

and one arrives at

$$
g=\frac{\sin \left(k_{y} L / 2\right)}{\pi} \frac{\sin (k R)}{k R}, \quad \rightarrow \quad Z \propto \frac{1}{R^{2}} .
$$

Note that (1) among sources collapsed along the tether, Estes [1988] model has, coincidentally, the right length dependence and (2) the scaling may be read as $Z \propto A^{-1}$ ( $A \equiv$ contactor area).

Using (21) in (15') with $k^{2}=k_{\mathrm{SE}}^{* 2}+k_{1}^{2}=k_{1}^{2} k_{e}^{2} /\left(k_{x}^{2}\right.$ $\left.-k_{\text {pe }}^{2}\right)$, setting $\sin ^{2}\left(k_{y} L / 2\right) \simeq \sin ^{2}(k R) \simeq 1 / 2, k_{\mathrm{UH}} \simeq$ $k_{p e}+k_{e}^{2} / 2 k_{p e}$ and calling $k_{y} / k_{x} \equiv u$, we find

$$
\begin{aligned}
& Z_{\mathrm{SE}}= \frac{2 V}{\pi R^{2} \Omega_{e}^{2}} \int_{k_{p e}}^{k_{\mathrm{UH}}} \frac{d k_{x}}{k_{x}}\left(\frac{k_{x}^{2}-k_{\mathrm{pe}}^{2}}{k_{\mathrm{UH}}^{2}-k_{x}^{2}}\right)^{1 / 2} \\
& \times \int_{0}^{\infty} \frac{d u}{\left(1+u^{2}\right)^{3 / 2}} \simeq \frac{V}{2 \omega_{p e}^{2} R^{2}} \\
&=\frac{\pi V}{\omega_{\mathrm{pe}}^{2}}\left(\frac{1}{A_{\mathrm{cat}}}+\frac{1}{A_{\mathrm{an}}}\right)
\end{aligned}
$$


with $A_{\text {cat }}=A_{\text {an }}=4 \pi R^{2}$. The only previous result $\left(Z_{\mathrm{SE}}=2 V^{2} / b^{3} \omega_{p e}^{3}\right)$ corresponds to BO's model, which underestimates $Z_{\mathrm{SE}}$, for $b \sim R$, by a typically small factor, $4 V / R \omega_{p e} \sim 1 / R(\mathrm{~mm})$. When (21) is used in (16'), only values $k_{x} \sim k_{\mathrm{LH}}$ contribute to the integral. We may then set $k_{x} / k_{e} \rightarrow 0$, integrate throughout the range $k_{\mathrm{LH}} \leq k_{x}<\infty$, and write $k^{2}=k_{\mathrm{FM}}^{* 2}+k_{\perp}^{2} \simeq k_{\perp}^{2}$ to find

$$
\begin{aligned}
Z_{\mathrm{FM}} & \simeq \frac{2 V}{\pi R^{2} \omega_{p e}^{2}} \int_{k_{\mathrm{LH}}}^{\infty} \frac{d k_{x}}{k_{x}} \frac{k_{e}}{\left(k_{x}^{2}-k_{\mathrm{LH}}^{2}\right)^{1 / 2}} \\
& \times \int_{0}^{\infty} \frac{d u}{\left(1+u^{2}\right)^{3 / 2}}=\frac{V}{\omega_{p e} \omega_{p i} R^{2}} \\
& =\frac{2 \pi V}{\omega_{p e} \omega_{p i}}\left(\frac{1}{A_{\mathrm{cat}}}+\frac{1}{A_{\mathrm{an}}}\right) .
\end{aligned}
$$

No previous analytical formula for $Z_{\mathrm{FM}}$ was available. Note the simple results $(22)$ and $(23)$.

Note also that $Z_{\mathrm{FM}}$ is not influenced by hot-plasma effects, despite concerns to that effect raised earlier (see BO, Hastings et al. [1988], Donohoue et al. [1991]). This is a pleasant side effect of the fact that a contactor will have a nonvanishing characteristic length along the tether, making the contribution from values, say, $k_{x}, k_{y}<10 k_{\mathrm{LH}}$ (approximately inverse electron gyroradius), dominant in the cold-plasma double integral in (16'). Hot effects on SE waves suggest the energy in such waves should experience heavy collisionless damping [Hastings et al., 1988]; the small value found here for the ratio $Z_{\mathrm{SE}} / Z_{\mathrm{FM}}=m_{e} / 2 m_{i} \sim 10^{-5}$ further suggests that such energy is negligible. Note also the ratio

$$
\begin{gathered}
\frac{Z_{\mathrm{A}}}{Z_{\mathrm{FM}}}=2\left(\frac{m_{i}}{m_{e}}\right)^{1 / 2} \frac{R^{2} \Omega_{i}^{2}}{V_{\mathrm{A}} V} \ln \left(2 e^{\gamma-1} \frac{\Omega_{i} L}{V}\right) \\
\simeq\left[\frac{n_{e}\left(\mathrm{~cm}^{-3}\right)}{3.6 \times 10^{5}}\right]^{1 / 2}\left[\frac{R(\mathrm{~m})}{13}\right]^{2} \ln \frac{L(\mathrm{~km})}{0.028}
\end{gathered}
$$

taken from (19') and (23); $n_{e}$ is electron density.

We now check our having assumed $k_{x}, k_{z}$ to be large compared with inverse perpendicular contactor lengths, for the integrals in (15') and (16'). This is clear for the $\mathrm{SE}$ branch, where we have $k_{z}=k_{\mathrm{SE}}^{*} \sim k_{x} \simeq \omega_{p e} / V \sim$ $1 / 0.2 \mathrm{~mm}$. The FM case is more involved. Although we do have $k_{x} \geq \omega_{\mathrm{LH}} / V \sim 1 / 20 \mathrm{~cm}$, which is comparatively large, $k_{z} \equiv k_{\mathrm{FM}}^{*}$ is smaller by a factor of order $k_{\mathrm{LH}} / k_{e}=\left(m_{e} / m_{i}\right)^{1 / 2}$ for $k_{x} \sim \omega_{\mathrm{LH}} / V$. However, in a proper model of $g$ such as given by $(21), k_{z}$ appears only in $k_{\text {per }}=\left(k_{x}^{2}+k_{z}^{2}\right)^{1 / 2}>k_{x}$, and our assumption holds.

Nonetheless, consider uniform current divergence on the surface of a rotational, prolate ellipsoid of major and minor semi-axes $a$ and $b$ along and perpendicular to $\mathbf{B}_{0}$ respectively. A straightforward development gives the result

$$
\begin{aligned}
g= & \frac{\sin \left(k_{y} L / 2\right)}{\pi} \frac{2 \int_{0}^{1} d \eta\left(1-\eta^{2} e^{2}\right)^{1 / 2}}{\left(1-e^{2}\right)^{1 / 2}+(\arcsin e) / e} \\
& \times \cos \left(k_{z} a \eta\right) J_{0}\left[k_{\perp} b\left(1-\eta^{2}\right)^{1 / 2}\right]
\end{aligned}
$$

where $e=\left(1-b^{2} / a^{2}\right)^{1 / 2}$. For $b=a \equiv R(e=0)$ one recovers (21). For an opposite case, $V / \omega_{\mathrm{LH}}<<b<<$ $a<<V / \Omega_{i}$, in the FM branch, one gets

$$
\begin{gathered}
g=\frac{\sin \left(k_{y} L / 2\right)}{\pi}\left[J_{0}^{2}\left(\frac{k_{1} b}{2}\right)-J_{1}^{2}\left(\frac{k_{\perp} b}{2}\right)\right] \\
\simeq \frac{\sin \left(k_{y} L / 2\right)}{\pi} \frac{4}{\pi} \frac{\sin \left(k_{\perp} b\right)}{k_{\perp} b} .
\end{gathered}
$$

Clearly, the impedance then reads

$$
Z_{\mathrm{FM}} \simeq \frac{16}{\pi^{2}} \frac{V}{b^{2} \omega_{p e} \omega_{p i}}=\frac{a}{b} \frac{8 V}{\omega_{p e} \omega_{p i}}\left(\frac{1}{A_{\text {cat }}}+\frac{1}{A_{\text {an }}}\right)
$$

which is much larger than the value for the spherical case as given by (23). Note, however, that the actual divergence might be highly nonuniform.

\section{Bare Tether Impedance}

It has been shown recently that a tether could work efficiently without an anodic contactor, by collecting electrons along certain length of its anodic end, if bare [Sanmartín et al., 1993]. If actually bare along its entire length, a (generator) tether was found to be optimal if positively biased over a length $l_{a} \simeq \frac{1}{7} L$. The current $\tilde{I}(y)$ would vanish at $y=L / 2$ and reach a nearly constant value $I_{s}$ at $y=L / 2-l_{a}$, with $d \tilde{I} / d y \propto-\left(y-\frac{1}{2} L+l_{a}\right)^{1 / 2}$ (Figure 3 ). If $b$ is the collecting radius in the $x, z$ plane and the cathodic contactor is a sphere of radius $R$, we then have

$$
\begin{gathered}
\nabla \cdot \mathbf{j}_{s}=\frac{I_{s} \delta\left(\left|\mathbf{r}+\frac{1}{2} L \mathbf{1}_{y}\right|-R\right)}{4 \pi R^{2}}-I_{s} \frac{\left(y-\frac{1}{2} L+l_{a}\right)^{1 / 2}}{\frac{2}{3} l_{a}^{3 / 2}} \\
\quad \times \frac{\delta\left(r_{\text {per }}-b\right)}{2 \pi b}\left[h\left(y-\frac{L}{2}+l_{a}\right)-h\left(y-\frac{L}{2}\right)\right]
\end{gathered}
$$

where $h$ is the unit step function. For $k_{y}>0$ we find

$$
\begin{aligned}
g & =\frac{\sin k R}{2 \pi i k R} e^{i k_{y} L / 2}-\frac{3 J_{0}\left(k_{\mathrm{per}} b\right)}{4 \pi k_{y} l_{a}} e^{-i k_{y} L / 2} \\
& \times\left[1-e^{i k_{y} l_{a}} \frac{C_{2}\left(k_{y} l_{a}\right)-i S_{2}\left(k_{y} l_{a}\right)}{\left(2 k_{y} l_{a} / \pi\right)^{1 / 2}}\right],
\end{aligned}
$$

with Fresnel integrals $\left[C_{2}(s), S_{2}(s)\right] \equiv \int_{0}^{s}\{\cos u, \sin u\}$ $\times d u /(2 \pi u)^{1 / 2}$, and 

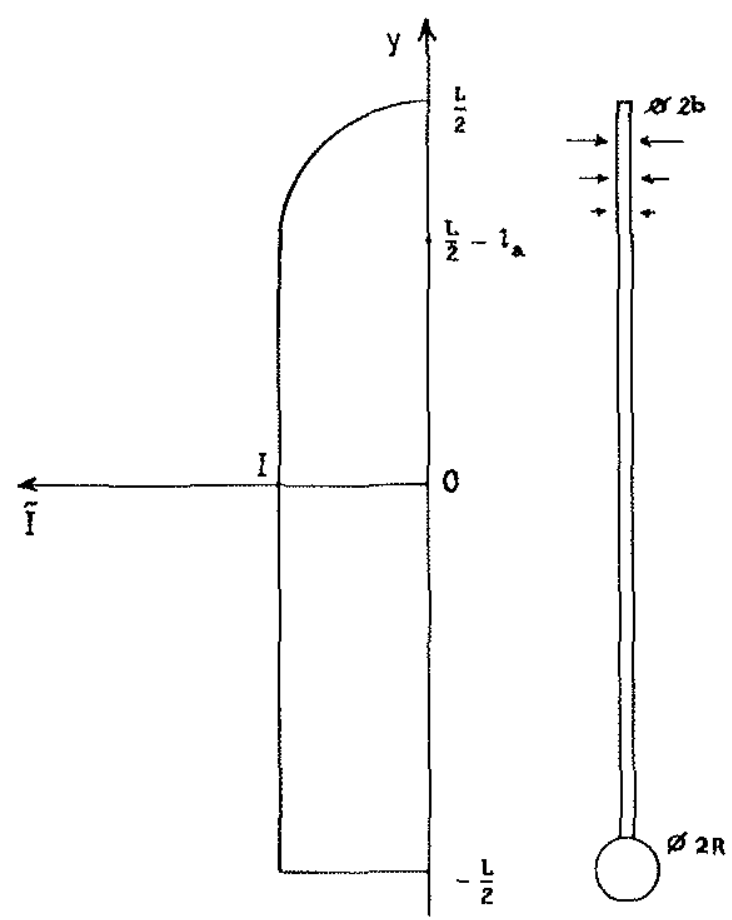

Figure 3. Anodeless, bare tether collecting electrons at its upper (anodic) end, over a positively biased length $l_{a}(\simeq L / 7)$; the bias vanishes at height $L / 2-l_{a}$.

$$
\begin{gathered}
|g|^{2}=\left(\frac{\sin k R}{2 \pi k R}\right)^{2}+\frac{J_{0}^{2}\left(k_{\mathrm{per}} b\right) F\left(k_{y} l_{a}\right)}{4 \pi^{2}}+\frac{3 J_{0}\left(k_{\mathrm{per}} b\right)}{4 \pi^{2}} \\
\times \frac{\sin k R}{k R}\left[g_{1}\left(k_{y} l_{a}\right) \cos \left(k_{y} L\right)+g_{2}\left(k_{y} l_{a}\right) \sin \left(k_{y} L\right)\right], \quad(27) \\
F(s)=\frac{9 \pi}{8 s^{3}}\left\{\left[S_{2}(s)-\left(\frac{2 s}{\pi}\right)^{1 / 2} \sin s\right]^{2}\right. \\
\left.+\left[C_{2}(s)-\left(\frac{2 s}{\pi}\right)^{1 / 2} \cos s\right]^{2}\right\} \\
g_{1}(s)=\left(\pi / 2 s^{3}\right)^{1 / 2}\left[S_{2}(s) \cos s-C_{2}(s) \sin s\right], \\
g_{2}(s)=\left(\pi / 2 s^{3}\right)^{1 / 2}\left[C_{2}(s) \cos s+S_{2}(s) \sin s\right]-1 / s .
\end{gathered}
$$

For the Alfvén branch and $k R, k_{\text {per }} b<<1$, we have $g \rightarrow g\left(k_{y}\right),(27)$ becoming

$$
\begin{gathered}
4 \pi^{2}|g|^{2} \simeq 1+F\left(k_{y} l_{a}\right)+3 g_{1}\left(k_{y} l_{a}\right) \cos \left(k_{y} L\right) \\
+3 g_{2}\left(k_{y} l_{a}\right) \sin \left(k_{y} L\right)
\end{gathered}
$$

for use in $\left(19^{\prime \prime}\right)$. Note that if $k_{y} l_{a} \rightarrow 0$, we recover $|g|^{2}=\sin ^{2}\left(k_{y} L / 2\right) / \pi^{2}$. At large $s \equiv k_{y} l_{a}$ we have $F(s)=0\left(s^{-2}\right), g_{1}(s)=0\left(s^{-3 / 2}\right), g_{2}(s)=0\left(s^{-1}\right)$, and $4 \pi^{2}|g|^{2}+1$. We can thus integrate separately ranges $k_{y}<k_{m}$ and $k_{y}>k_{m}$, with

$$
7 / L \simeq 1 / l_{a} \ll k_{m} \ll \Omega_{i} / V .
$$

- Equation(19") can then be written as

$$
\begin{gathered}
\frac{c^{2} Z_{\mathrm{A}}}{V_{\mathrm{A}}} \simeq \int_{k_{m}}^{\infty} \frac{d k_{y}}{k_{y}} \frac{\left(k_{i}^{2}+k_{y}^{2}\right)^{1 / 2}-k_{y}}{k_{i}} \\
+\int_{0}^{k_{m} l_{a}} d s \frac{1+F(s)+3 g_{1}(s) \cos 7 s+3 g_{2}(s) \sin 7 s}{s}
\end{gathered}
$$

leading to an impedance

$$
\begin{gathered}
Z_{\mathrm{A}}=\frac{V_{\mathrm{A}}}{c^{2}} \ln \left(2 e^{\alpha-1} \Omega_{i} \frac{L}{V}\right), \\
\alpha \equiv \int_{0}^{1}\left[1+F(s)+3 g_{1}(s) \cos 7 s\right] \frac{d s}{s} \\
+\int_{1}^{\infty}\left[F(s)+3 g_{1}(s) \cos 7 s\right] \frac{d s}{s} \\
+\int_{0}^{\infty} 3 g_{2}(s) \sin 7 s \frac{d s}{s}-\ln 7 \simeq 4.00 .
\end{gathered}
$$

For the FM branch we may take $k R$ large. Then the last term in (27) makes a negligible contribution to the impedance $z_{\mathrm{FM}}$ as shown in (16), while the first term yields one half the value given by (23), in agreement with the general result that anodic and cathodic contactors make independent contributions to the FM impedance [Donohue et al., 1991]. For the middle term we can set $k_{y} \sim 1 / l_{a} \ll \omega_{\mathrm{L}, \mathrm{H}} / V<k_{x}$ and $k_{z}=k_{\mathrm{FM}}^{*} \ll \omega_{\mathrm{LH}} / V \sim k_{x}$, thus writing $k_{\perp} \simeq k_{x}$, $k_{\text {per }} \sim k_{x}$. If, additionally, $k_{\text {per }} b$ is large, we arrive at

$$
\begin{gathered}
Z_{\mathrm{FM}}=\frac{V / 2}{R^{2} \omega_{p e} \omega_{p i}}+\frac{2 V}{\pi \omega_{p e}^{2} b l_{a}} \int_{k_{\mathrm{LH}}}^{\infty} \frac{d k_{x}}{k_{x}} \frac{k_{e} \beta}{\left(k_{x}^{2}-k_{\mathrm{LF}}^{2}\right)^{1 / 2}} \\
=\frac{2 \pi V}{\omega_{p e} \omega_{p i}}\left(\frac{1}{A_{\mathrm{cat}}}+\frac{\beta}{A_{\text {an }}}\right) \\
A_{\text {an }} \equiv 2 \pi b l_{a}, \quad \beta \equiv \int_{0}^{\infty} \frac{F(s) d s}{\pi} \simeq 1.12 .
\end{gathered}
$$

Note that written in terms of areas, (23) and (29) read nearly the same.

Actually, condition $b \gg 1 / k_{\text {per }} \sim 20 \mathrm{~cm}$ will not be satisfied if $b$ is the actual radius of the tether; for $k_{\text {per }} b$ small we would have $Z_{\mathrm{FM}} \simeq 2 \pi V / \omega_{p e} \omega_{p i} A_{\text {cat }}$, or one half the value given in (23). At this point we raise the question of what are the lengths really characterizing $\nabla \cdot \dot{j}_{s}$. Estes [1988] suggested that the plasma cloud emitted by an active contactor might extend the effective dimensions well beyond the dimensions of the contactor itself; Donohue et al. [1991] further suggested that the sheath radius should be an effective radius for passive contactors.

The basic argument underlying these suggestions is the need to account for nonlinear effects, which are essential for a self-consistent analysis. A crude recipe is to consider the entire nonlinear region around the contac- 
tor as part of the contactor itself. For the anodic part of a bare tether, which collects electrons as a cylindrical Langmuir probe in the orbital-motion- limited regime, the faraway electric potential decays as the inverse of distance to tether [Laframboise, 1966]. Then, for typical electron temperature $T_{e} \simeq 0.1 \mathrm{eV}$, tether radius $(\sim 1$ $\mathrm{mm}$ ) and anodic bias (hundreds of volts), the average $b$ would be of order of meters, large, indeed, compared with $1 / k_{\text {per }}$.

The above argument suggests that nonlinear effects would adjust contactor areas to effective values

$$
A_{\text {cat }} \sim I_{s} / j_{t h} \sim A_{\text {an }},
$$

where $j_{t h}$ is the (unperturbed) electron random current density, $j_{t h} \equiv \frac{1}{4} e n_{e}\left(8 T_{e} / \pi m_{e}\right)^{1 / 2}$, with $e \equiv$ electron charge. Using (30) in our previous (23) or (29) and using $m_{i} V^{2} \simeq 8.9 \mathrm{eV}$ then yields a very simple result

$$
Z_{\mathrm{FM}} I_{s} \sim \frac{4 \pi V j_{t h}}{\omega_{p e} \omega_{p i}}=\left(\frac{m_{i} V^{2} T_{e}}{2 \pi e^{2}}\right)^{1 / 2} \simeq 0.38 \mathrm{~V} .
$$

Note that the power radiated in the FM branch, $Z_{\mathrm{FM}} I_{s}^{2}$, will increase only linearly (rather than quadratically) with current. FM radiation will dominate Alfvén radiation, except at large currents, when the effective contactor area and effective radius $R$ is large; this is reflected in (24), where $Z_{\mathrm{A}} / Z_{\mathrm{FM}}$ scales as $R^{2}$. A $0.5 \mathrm{~km}$ long (the so-called PMG) tether, flown in June 1993, reached a current $I_{s} \simeq 0.3 \mathrm{~A}$ at the maximum density $n_{e} \simeq 10^{6} \mathrm{~cm}^{-3}$, yielding $R \simeq 1.54 \mathrm{~m}$ in $(30) ;(31)$ and (24) then give $Z_{\mathrm{FM}} \simeq 1.27 \Omega$ and $Z_{\mathrm{A}} \simeq 0.086 \Omega$. For the aborted tethered satellite system (TSS) 1 experiment the effective area was probably the actual physical surface of the passive anode (a sphere of radius 0.8 $\mathrm{m}$ ), the current in (30) being of purely thermal origin, $I_{s} \simeq 0.066 \mathrm{~A}$ at $n_{e} \simeq 10^{6} \mathrm{~cm}^{-3}$. Equation (31) then yields $Z_{\mathrm{FM}} \simeq 5.7 \Omega$, a result also directly obtained from (23). Since the deployed tether length was $L \simeq 300 \mathrm{~m}$, one finds $Z_{\mathrm{A}} / Z_{\mathrm{FM}} \simeq 0.015$ in (24).

\section{Summary of Results}

We have used the potential $\phi$ for the longitudinal part of the electric field of a wave and conditions particular to low Earth orbits in discussing the five branches $\omega(k, \theta)$ of the dispersion relation for a cold, magnetized plasma. No emission of fast extraordinary or ordinary waves is possible. Slow extraordinary (SE) and fast magnetosonic (FM) emission occurs near $k \rightarrow \infty$ asymptotes, with $\mathbf{E} \simeq-\nabla \phi$. Alfvén $(A)$ emission occurs in a peculiar regime, arising from condition

$$
V_{\mathrm{A}}^{2} \ll V^{2} m_{i} / m_{e},
$$

with $\mathbf{E} \simeq-\nabla_{\perp} \phi$. The discussion clarifies or corrects some published results on group velocities, emission bands, and emission of whistlers or outside contactors.
The $\phi$ formalism appears useful for future work on a number of standing issues. The formalism has been used here to determine the impedance of an orbiting conductor.

For tethers we found that usual models, having vanishing contactor length along the tether $\left(L_{y}=0\right)$, yield values $Z_{\mathrm{FM}}$ (and $Z_{\mathrm{SE}}$ ) heavily dependent on the model. A proper model requires $L_{y} \neq 0$ and this yields

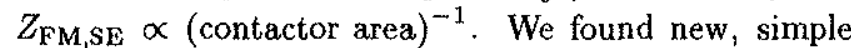
formulas for $Z_{\mathrm{SE}}$ (see (22)), so small that power radiated into this strongly damped branch should be negligible, and $Z_{F M}(23)$ unaffected by hot-plasma effects. An anodeless, bare tether of equal area, has a very similar $Z_{\mathrm{FM}}(29)$. Nonlinear effects might make contactor area proportional to current $I_{s}$, and this would lead to an extremely simple result, (31). We have also found $Z_{\mathrm{FM}}$ for ellipsoidal contactors (see (26)) and $Z_{\mathrm{A}}$ for contactors with $L_{y} \neq 0$ but with vanishing lengths perpendicular to the tether (see $\left(19^{\prime \prime}\right)$ ), particularized to a bare tether in $(28)$.

\section{Appendix}

Consider an $(x, z)$ surface contactor with polygonal contour, as in the rectangular model for (17) by Hast. ings et al. [1988]. We then have

$$
\begin{gathered}
g_{\mathrm{per}}=\int_{s_{\mathrm{m}}}^{s_{\mathrm{M}}} e^{-i k_{\mathrm{per}} s} \frac{d s}{A_{c}} w_{c}(s) \\
=\int_{s_{n 2}}^{s_{\mathrm{M}}} e^{-i k_{\mathrm{per}}} \frac{d s}{A_{c}} \frac{d w_{c} / d s}{i k_{\mathrm{per}}}
\end{gathered}
$$

where $A_{c}$ is area and $w_{c}(s)$ is full width at given $s$; we used a partial integration and the vanishing of $w_{c}(s)$ at both $s_{m}$ and $s_{M}$ (Figure $4 \mathrm{a}$ ). Since $d w_{c} / d s$ is piecewise constant, $g_{\text {per }}$ is a sum of straightforward integrals, yielding $g_{\text {per }} \propto 1 / A_{c} k_{\text {per }}^{2} \propto 1 / A_{c}, Z \propto 1 / A_{c}^{2}$, in agreement with the result in $(20)$.

If the contour is a smooth curve, as in the BO model, $d w_{\mathrm{c}} / d s$ diverges at both $s_{m}$ and $s_{\mathrm{M}}$ (Figure 4b). A range $\Delta s \sim 1 / k_{\text {per }}$ around each extreme then gives dominant contributions of order $1 / k_{\mathrm{per}}^{3 / 2}$ to (A1); within such range we have $d w_{c} / d s \sim(R / \Delta s)^{1 / 2}$, where $R \sim A_{c}^{1 / 2}$ is the radius of curvature of the contour. This yields $g_{\text {per }} \propto \Delta s(R / \Delta s)^{1 / 2} / A_{c} k_{\text {per }} \propto 1 / A_{c}^{3 / 4} k_{\text {per }}^{3 / 2} \propto$ $1 / A_{c}^{3 / 4}, Z \propto 1 / A_{c}^{3 / 2}$, as in (20).

For models with line rather than surface contactors we would have

$$
g_{\mathrm{per}}=\int_{s_{m}}^{s_{\mathrm{M}}} e^{-i k_{\mathrm{per}} s} \frac{d s}{L_{c}} \frac{d l_{c}}{d s},
$$

where $l_{c}(s)$ is the length lying between $s_{m}$ and a given $s$, and $L_{c}$ is total length (Figures $4 \mathrm{a}$ and $4 \mathrm{~b}$ ). For a polygonal (open or closed) curve, $d l_{c} / d s$ is piecewise constant, yielding $g_{\mathrm{per}} \propto 1 / L_{c} k_{\mathrm{per}} \propto 1 / L_{c}, Z \propto 1 / L_{c}^{2}$. 

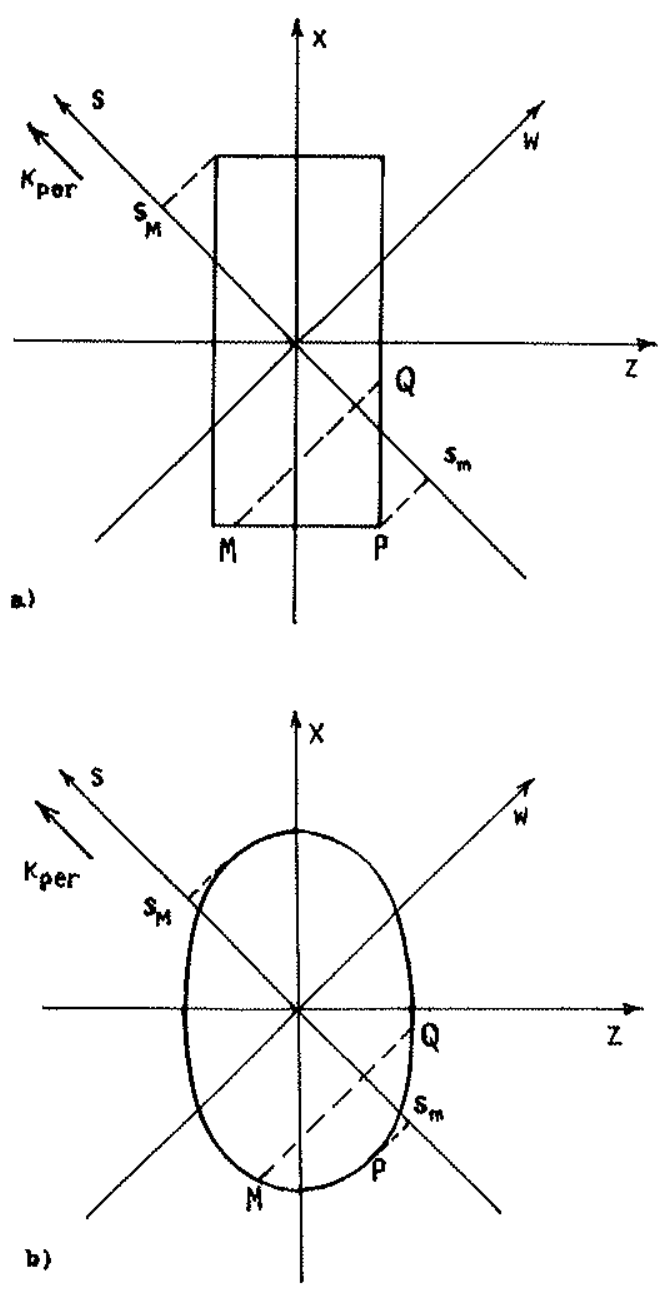

Figure 4. Cross section of contactor with vanishing length along tether and (a) polygonal, (b) smooth shape. For a surface contactor, $w_{c}(s)$ represents the width $M Q$; for a line contactor, $l_{c}(s)$ represents the length of the curve MPQ.

For a smooth curve, $d l_{c} / d s$ diverges at $s_{m}$ and $s_{M}$; if $R \propto L_{c}$ is radius of curvature, (A2) gives $g_{\text {per }} \propto$ $\Delta s(R / \Delta s)^{1 / 2} / L_{c} \propto 1 / L_{c}^{1 / 2} k_{\operatorname{per}}^{1 / 2} \propto 1 / L_{c}^{1 / 2}, Z \propto 1 / L_{c}$. This explains the results in $(20)$ for the Estes [1988] and ring models.

Acknowledgments. This work was supported by the Comision Interministerial de Ciencia y Tecnología of Spain (project ESP92 0989-E).

The Editor thanks B.E. Gilchrist and D.J. Donohue for their assistance in evaluating this paper.

\section{References}

Akhiezer, A.I., I.A. Akhiezer, R.V. Polovin, A.G. Sitenko, and K.N. Stepanov, Plasma Electrodynamics, vol.1, Pergamon, New York, 1975.

Banks, P.M., P.R. Williamson, and K.L. Oyama, Electri- eal behavior of a shuttle electrodynamic tether system (SETS), Planet. Space Sci., 29, 139, 1981.

Barnett, A., and S. Olbert, Radiation of plasma waves by a conducting body moving through magnetized plasma, $J$. Geophys. Res., 91, 10,117, 1986.

Dobrowolny, M., and P. Veltri, MHD power radiated by a large conductor in motion through a magnetoplasma, Nuovo Cimento Soc. Ital. Fis. C., 9, 27, 1986.

Donohue, D.J., T. Neubert, and P.M. Banks, Estimating radiated power from a conducting tethered satellite system, J. Geophys. Res., 96, 21,245, 1991.

Drell, S.D., H. M. Foley, and M. A. Ruderman, Drag and propulsion of large satellites in the ionosphere: An Alfvén propulsion engine in space, J. Geophys. Res., 70, 3131, 1965.

Estes, R.D., Alfvén waves from an electrodynamic tethered satellite system, J. Geophys. Res., 99, 945, 1988.

Hastings, D.E., and J. Wang, The radiation impedance of an electrodynamic tether with end connectors, Geophys. Res. Lett., 14, 519, 1987.

Hastings, D.E., and J. Wang, Induced emission of radiation from a large space-station-like structure in the ionosphere, AIAA J., 27, 438, 1989.

Hastings, D.E., A. Barnett, and S. Olbert, Radiation from large space structures in low Earth orbit with induced alternating currents, J. Geophys. Res., 99, 1945, 1988.

Laframboise, J.G., Theory of spherical and cylindrical Langmuir probes in a collisionless, Maxwellian plasma at rest, Rep. 100, Univ. of Toronto, Inst. for Aerosp. Stud. Toronto, Canada, 1966.

McKenzie, J.F., Stationary MHD waves generated by a source in a moving plasma, $J$. Geophys. Res., 96, 9491, 1991.

Rasmussen, C.E., P.M. Banks, and K.J. Harker, The excitation of plasma waves by a current source moving in a magnetized plasma: The MHD approximation, $J$. Geophys. Res., 90, 505, 1985.

Rasmussen, C.E., P.M. Banks, and K.J. Harker, The excitation of plasma waves by a current source moving in a magnetized plasma: Two-dimensional propagation, $J$. Geophys. Res., 95, 10,459, 1990.

Sanmartín, J.R., M. Martínez-Sánchez and E. Ahedo, Bare wire anodes for electrodynamic tethess, $J$. Propul. Power, $9,353,1993$.

Stenzel, R.L., and J.M. Urrutia, Currents between tethered electrodes in a magnetized laboratory plasma, J. Geophys. Res., 95, 6209, 1990.

Urrutia, J.M., and R.L. Stenzel, Transport of current by whistler waves, Phys. Rev. Lett., 62, 272, 1989.

vom Stein, R., and F.M. Neubauer, Plasma wave field generation by the tethered satellite system, J. Geophys. Res., $97,10,849,1992$.

M. Martínez-Sánchez, Department of Aeronautics and Astronautics, Massachusetts Institute of Technology, Cambridge, MA 02139. (e-mail:mmart@mit.edu)

J. R. Sanmartín, E.T.S.Ingenieros Aeronáuticos, Universidad Politécnica, Plaza Cardenal Cisneros 3, 28040-Madrid, Spain.

(Received April 20, 1994; revised October 13, 1994; accepted October 28, 1994.) 